\title{
Osteoma Left Mastoid Bone: A Case Report
}

\author{
*MA Chowdhury ${ }^{1}$, AS Majumder ${ }^{2}$, M Uddin $^{3}$ \\ $1 *$ Prof. Dr. M. Alamgir Chowdhury, Professor and Head, ENT- HNS, Anwer Khan Modern Medical College, Dhaka \\ ${ }^{2}$ Dr. Afroza Suraya Majumder, Assistant Professor, ENT - HNS, Anwer Khan Modern Medical College, Dhaka \\ ${ }^{3}$ Dr. Maskat Uddin, Medical Officer, ENT Deptt, Anwer Khan Modern Medical College, Dhaka \\ *Corresponding Author
}

Date of submission: 10.08.2016 Date of acceptance: 05.12.2016

\begin{abstract}
Osteoid ostemoa is benign bone tumor and third most common benign neoplasm of bone. Young male are frequently affected three times more than females. It can occur in any bone but most common in long bone such as femer and tibia. In routine ENT practice osteoma are infrequently found in temporal bones most commonly seen in external ear canal. Osteoid ostemoa in mastoid are rare. We present such a rare presentation of ostema arising form the left mastoid in a young female.
\end{abstract}

Key Words: Osteoma, Mastoid bone

\section{Introduction}

Ostemoas are benign tumor. They arises from cancellous bone. They are solitary often unilateral and usually arise spontaneously. These can present in the squamous part of temporal bone, mastoid, internal auditory meatus, the middle ear and other bone. ${ }^{1,2,3}$ In the literature only 130 cases has been reported between 1861 and 2004. ${ }^{3}$ They are usually asymptomatic but may enlarge to cause cosmetic deformity. The etiology in poorly understood and may be due to trauma, infection and herditrary. Computed tomography is the gold standard for diagnosis subsequently confirmed by histopathological diagnosis. Treatment should be surgical excision for cosmetic purpose or if there is any symptoms. $5,6,7$

\section{Case Report}

A 23 year old female patient otherwise normal presented with otolaryngologically asymptomatic swelling in left mastoid region for 5 years. It was found in shape painless, very hard, overlying skin is free and adherent to the underlying bone. There is

AKMMC J 2017; 8(1): 71-72 no history earache, hearing impairment and neurological deficit. She did not give any history of trauma. On examination she was found to have a $2 \mathrm{cmX} 2 \mathrm{~cm}$ solid nodule. Detail ENT examination including facial nerve foundations was normal. CT scan revealed focal thickness at the outer aspect of the left mastoid process (Figure-1). There was no bony exostosis in the external cannal. The was no other osteomas elsewhere in the skull.

CT scan revealeda focal thickness at the outer aspect of the left mastoid process.(Figure 1)

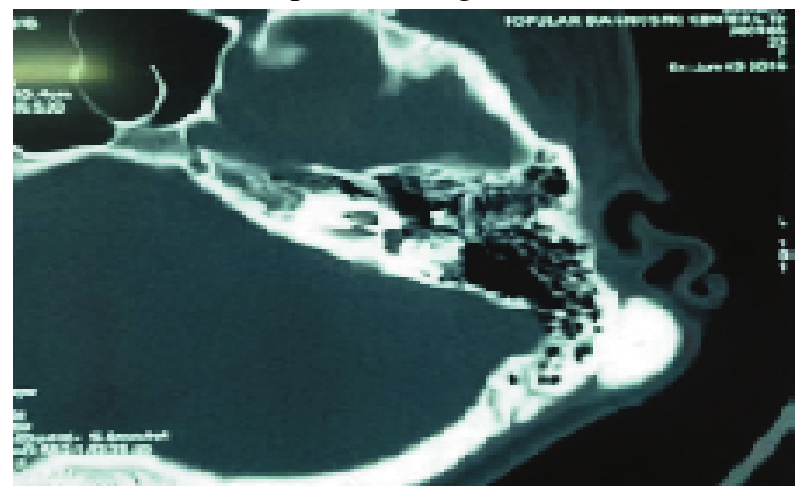

Figure 1: CT scan revealeda focal thickness at the outer aspect of the left mastoid process. 
AKMMC J $2017: 8(1)$

Surgical excision was bone under general anesthesia with help of hammer and chisel. Bone edge was smoothening by drilling. Histopathological report showed mastoid ostemoa. Post-operative period was excellent and she was fine in the post operative follow up.

\section{Discussion}

Mastoid osteomae is rare and common in young adult, Males are affected 3 thing more than female. 8 It usually grows as single nodule and grows form the gutter table of th mastoid cortex producing an external swelling. The exact pathology of osteomas are not well understood but they are thought to arise from preosseous connective tissue. Tehre are evidence that osteomas are of congenital nature. It may also occur as a part of syndrome like Gardners syndrome but have a prediliciton membranous bone such as mandible and maxilla that are commonly involved. ${ }^{9}$

Ostemoa of temporal bone is not uncommon but in mastoid is comparatively rare. It is a benign slow growing tumor, more common in female after puberty which is consistent with our case a female of 23 year. Our case has no history of trauma hearing impairment and infection, although in most cases of osteoid osteoma patient complains of inflammatory condition like pain that worsen at with and alleviated by using NSAIDS. Facial canal bony labyritnth or other surrounding structure may involved in osteoma. In our case it was on mastoid part of temporal bone as in CT scan finding. Complete surgical excision was done successfully for cosmetic reason in our case which is the common indication of surgery.
*MA Chowdhury, AS Majumder, M Uddin

\section{Conclusion}

We present here a comparatively rare case of osteoid ostemoa in young female at left mastoid for record with a caution that appropriate diagnosis with early surgical intervention is needed and it should be kept in mind as it may go up to intracranial.

\section{Conflict of interest: None}

\section{Reference}

1. HornigoldR, PearchBJ, Gleeson MJ. An osteoma of the middle ear presenting with the Tuliophenomenon. Skull Base 2003; 113-17.

2. LemmerlingMM, De Foer B, VandevyverV. Imaging of inflammatory \& infectious diseases in the temporal bone. Neuroimaging clinics of North America 2009; 19(3): 321-37.

3. ViswanathaB. Extercanlicular osteoma of the temporal bone.Ear nose throat J 2008: 87(7): 381-3.

4. J.S Gulia \& SY adav: mastoid osteoma: A case report. The internet journal of Head and Neck Surgery 2009: 3 (1): 22-23.

5. GungorA, AincikH, PoyrazogluE, SaglamO, CandanH. Mastoid osteoma: report of two cases. OtoINeuro-Otol 2004; 25-97.

6. Lee RE, BalkanyTJ, Giant mastoid osteoma with postoperative high frequency sensorineural hearing loss. Ear Nose Thorat J 2008: 87 (1): 23-5.

7 MagliuloG, PuliceG. Mastoid osteoma. Anotorhino laringol 2005; 32-.271-8.

8. https://en.wikipedia.org/wiki/Osteoid_osteoma

9. DasAK and KashyapRC. osteoma of the mastoid 